\title{
Primary central nervous system lymphoma treated with high-dose methotrexate and rituximab: A single-institution experience
}

\author{
K. INA LY ${ }^{1}$, LAURA L. CREW ${ }^{2}$, CARRIE A. GRAHAM $^{1}$ and MACIEJ M. MRUGALA ${ }^{1-3}$ \\ Departments of ${ }^{1}$ Neurology and ${ }^{2}$ Neurological Surgery, University of Washington Medical Centre, Seattle, WA 98195; \\ ${ }^{3}$ Fred Hutchinson Cancer Research Center, Seattle, WA 98109, USA
}

Received February 24, 2015; Accepted February 2, 2016

DOI: $10.3892 / \mathrm{ol} .2016 .4393$

\begin{abstract}
Rituximab (RTX) improves the outcome in patients with systemic diffuse large B-cell lymphoma (DLBCL), but its benefit in primary central nervous system lymphoma (PCNSL) is unclear. In the present study, a single-institution retrospective analysis was performed for 12 patients with newly diagnosed PCNSL treated with combined high-dose methotrexate (HD-MTX) and RTX. MTX was administered biweekly at $8 \mathrm{~g} / \mathrm{m}^{2} /$ dose until a complete response (CR) was achieved or for a maximum of eight doses. RTX was provided for a total of eight weekly doses at $375 \mathrm{mg} / \mathrm{m}^{2} / \mathrm{dose}$. Following a median of 11 cycles of MTX, the radiographic overall response rate was $91 \%$ and the CR rate was $58 \%$. A CR was achieved after a median 6 cycles of MTX. The median progression-free survival time was 22 months and the median overall survival time has not yet been attained. These results compare favorably to single-agent HD-MTX and suggest a role for immunochemotherapy in the treatment of PCNSL.
\end{abstract}

\section{Introduction}

Primary central nervous system lymphoma (PCNSL) is a predominantly extranodal non-Hodgkin's B-cell lymphoma (NHL) of the brain, spinal cord, leptomeninges and eyes, with an incidence of 0.47 cases per 100,000 person-years (1). Traditional therapies for systemic NHL, such as the cyclophosphamide, doxorubicin, vincristine and prednisone (CHOP) regimen, have proven to be ineffective in PCNSL, presumably due to poor drug penetration across the blood-brain barrier (BBB) (2). In the past, whole-brain radiation therapy (WBRT) was considered to be the first-line treatment for PCNSL due to high complete response rates (CRRs) of up to $90 \%$ (3). However, the median overall survival (OS) time and 2-year survival

Correspondence to: Dr Maciej M. Mrugala, Department of Neurology, University of Washington Medical Center, 1959 NE Pacific Street, Seattle, WA 98195, USA

E-mail: mmrugala@u.washington.edu

Key words: high-dose methotrexate, non-Hodgkin's lymphoma, primary central nervous system lymphoma, rituximab rate were only $12-17$ months and $28-40 \%$, respectively $(4,5)$. Subsequent trials demonstrated improved median OS times with combined WBRT and methotrexate (MTX)-based chemotherapy, prolonging the OS time from 11.5 months with WBRT alone (4) to 33-60 months with combined therapy (6-8). However, this regimen carries a significant risk of delayed neurotoxicity $(7,9)$, and therefore WBRT is usually delayed or avoided and reserved for cases of tumor recurrence. Several trials have investigated the efficacy of single-agent high-dose MTX (HD-MTX) and found variable CRRs ranging from $29-52 \%(10,11)$. There is now increasing evidence that MTX-based polychemotherapy is superior to HD-MTX alone, for instance, when MTX is combined with HD-cytarabine (Ara-C) or ifosfamide $(12,13)$. Furthermore, rituximab (RTX) is now frequently combined with single-agent HD-MTX or MTX-based polychemotherapy, based on observations that the addition of RTX results in improved response rates (14-18). The present study reports the experience of a single institution as a retrospective case series of 12 patients with newly diagnosed PCNSL treated with HD-MTX and RTX.

\section{Patients and methods}

Patient selection criteria. Following Institutional Review Board (IRB) approval by the University of Washington Medical Center (Seattle, WA, USA), a retrospective chart review was performed of patients with PCNSL at our institution between 2007 and 2011. Inclusion criteria consisted of an age $\geq 18$ years, a Karnofsky performance status (KPS) of $\geq 50 \%$, magnetic resonance imaging (MRI) evidence of disease and a histologically proven diagnosis. Exclusion criteria consisted of the presence of other types of malignancy and an immunocompromised state. All patients underwent staging with computed tomography (CT) of the chest, abdomen and pelvis, as well as an ophthalmological examination. In addition, all males underwent a testicular examination and ultrasound to exclude the presence of testicular lymphoma. A diagnosis of PCNSL was made by histopathological confirmation of either biopsy or cerebrospinal fluid (CSF) studies.

Treatment regimen. Patients underwent induction therapy with combined biweekly HD-MTX $\left(8 \mathrm{~g} / \mathrm{m}^{2} /\right.$ dose $)$ and weekly RTX $\left(375 \mathrm{mg} / \mathrm{m}^{2} /\right.$ dose). HD-MTX was administered intravenously over $4 \mathrm{~h}$ with anti-emetic premedication and with or without 
concurrent dexamethasone. All patients received aggressive hydration prior to and after HD-MTX infusion (sterile water $+150 \mathrm{mEq} / \mathrm{l}$ of $\mathrm{NaHCO} 3+20 \mathrm{mEq} / \mathrm{l}$ of $\mathrm{KCl}$ infused at $150-200 \mathrm{cc} / \mathrm{h}$ over $4 \mathrm{~h}$ ) to maintain a urine output of $\geq 100 \mathrm{ml} / \mathrm{h}$ and a urinary $\mathrm{pH}$ of $>7$, until serum MTX levels had decreased to $\leq 0.1 \mu \mathrm{M} / 1$. Serum MTX levels were monitored daily. Leucovorin rescue was initiated $24 \mathrm{~h}$ after completion of MTX infusion and administered at $25 \mathrm{mg}$ every $6 \mathrm{~h}$ until serum MTX levels were $\leq 0.1 \mu \mathrm{M} / 1$. Leucovorin dosing was increased in cases of slow MTX clearance. All patients were instructed to continue leucovorin at $25 \mathrm{mg}$ orally every $6 \mathrm{~h}$ for 2 days following discharge. HD-MTX during induction was administered until a CR was achieved by MRI criteria. In the absence of a CR, MTX was administered for a maximum of eight biweekly doses. RTX was provided for a total of eight weekly doses.

Responders to the induction regimen received consolidation treatment with 2 additional cycles of biweekly HD-MTX, followed by maintenance treatment with monthly HD-MTX for up to 12 months. The duration of maintenance treatment was primarily limited by drug toxicity according to the National Cancer Institute Common Toxicity Criteria (version 4.0; http://evs.nci.nih.gov/ftp1/CTCAE/CTCAE_ 4.03_2010-06-14_QuickReference_5x7.pdf).

Endpoints and response criteria. The primary end point was radiographic response, assessed by contrast-enhanced MRI every 2 cycles during induction and every 2 months thereafter.

Response was classified according to standard radiographic criteria as a CR, partial response (PR), stable disease (SD) or progressive disease (PD). A CR was defined as complete resolution of all contrast-enhanced lesions. A PR was defined as a $>50 \%$ reduction in size of contrast-enhancing lesions, and PD was defined as an increase in size by $>25 \%$. All other situations were classified as SD.

OS time was measured as the time between the pathological diagnosis and mortality or the time of last follow-up. Progression-free survival (PFS) time was defined as the time between the start of treatment and the day of radiographic progression.

\section{Results}

Demographics. A total of 12 patients were included in the present study ( 6 females and 6 males). The median patient age at the time of diagnosis was 62.5 years (range, 19-78 years). The median KPS was $80 \%$ (range, $50-100 \%$ ). Patient characteristics are listed in Table I.

Neurological manifestations. The most common symptoms were cognitive dysfunction $(n=5)$, including word-finding difficulties and altered mental status, and ataxia and incoordination $(n=4)$. Furthermore, 3 patients experienced vision changes and focal weakness. Less commonly observed symptoms consisted of headaches $(n=2)$, sensory changes $(n=2)$ and seizures $(n=1)$.

Tissue diagnosis and imaging. Tissue diagnosis was made either by biopsy $(n=10)$, resection $(n=1$; case 4$)$ or CSF analysis $(n=1$; case 9). Biopsy alone was diagnostic in $83 \%$ of patients $(n=10)$ and revealed diffuse large B-cell lymphoma (DLBCL), based on characteristic cellular morphology and antigen expression profile, including positivity for CD19, CD20 and CD45. CSF analysis in case 9 showed a clonal population of abnormal B cells. In total, 2 patients underwent a biopsy after non-diagnostic flow cytometry and cytology from CSF analysis (cases 3 and 6).

All patients underwent MRI of the brain and entire spine. Characteristic imaging findings included homogenously enhancing periventricular white matter lesions with associated diffusion restriction, although exceptions to these features were observed as well. Systemic involvement of lymphoma was excluded in 10 subjects by CT of the chest, abdomen and pelvis. Cases 2 and 4 underwent whole-body positron emission tomography (PET)-CT instead of CT of the chest, abdomen and pelvis. CT in case 9 revealed pulmonary nodules, but subsequent PET-CT excluded ${ }^{18}$ F-fluorodeoxyglucose avidity. None of the patients presented with intra-ocular involvement of lymphoma.

Treatment and treatment response. A total of 109 cycles of MTX were administered (104 induction and 5 maintenance cycles), with a mean of 8.6 cycles and a median of 11 cycles (range, 2-15 cycles).

The most commonly encountered side effects were fatigue $(n=4)$, anemia, neutropenia, nausea and renal dysfunction $(n=3$ each). While 2 individuals experienced transient transaminitis, 1 patient presented with thrombocy topenia and 1 with an RTX-related infusion reaction. Grade 3 toxicities were identified in 2 patients: Case 7 was affected by severe mucositis, febrile neutropenia, anemia and thrombocytopenia, requiring transfusion of blood products; case 5 was also transfused due to anemia. No toxicity was reported in 1 patient (case 11) and data was unavailable for another (case 9). No grade 4 toxicities were observed.

Salvage therapy was administered to 7 patients; 4 of these (cases 1, 4, 8 and 12) received intrathecal Ara-C alone or in combination with HD-MTX. Case 2 was re-challenged with 8 cycles of RTX and 1 cycle of temozolomide (TMZ), followed by gamma-knife stereotactic radiosurgery and WBRT. Case 10 received HD-MTX as salvage treatment prior to undergoing autologous peripheral blood stem cell transplantation (ASCT). Two additional patients (cases 11 and 12) underwent ASCT after achieving a CR.

The mean overall response rate (ORR) to treatment was $91.7 \%$ (11 out of 12 patients). A CR was achieved in 7 patients $(58.3 \%)$ and a PR in 4 patients $(33.3 \%)$. Survival data was not available for case 9. A median of 6 cycles of MTX (range, 4-19 cycles) had to be administered to achieve a CR (Fig. 1). In total, 3 patients (cases 1, 2 and 10) had recurrent disease after a median PFS time of 22.1 months (range, 6-27.7 months; Fig. 2). Seven patients did not experience disease progression after achieving a $\mathrm{CR}$ or $\mathrm{PR}$ (cases 3-8 and 11). At the time of data analysis, 10 out of 12 patients were alive after a median time of 8.5 months (range, 2.9-17.9 months) following treatment response. Case 2 succumbed to complications associated with pneumonia and a pulmonary embolus. Case 12 underwent an ASCT as part of salvage therapy and succumbed to respiratory failure associated with lymphocytic alveolitis and a drug reaction with eosinophilia and systemic symptoms. 


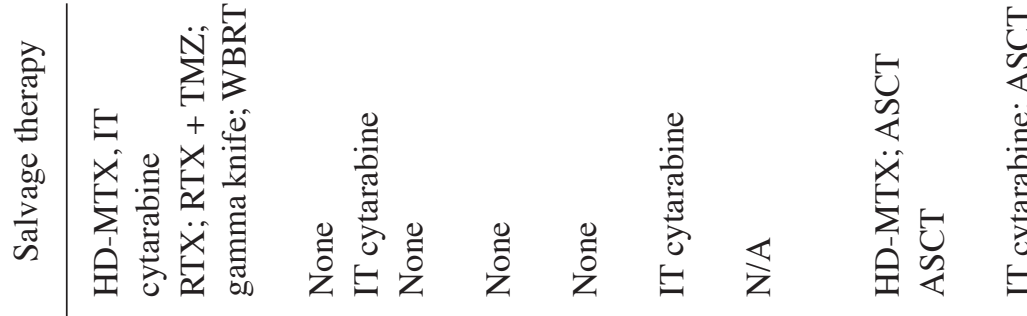

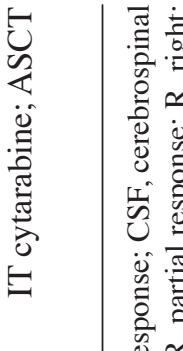

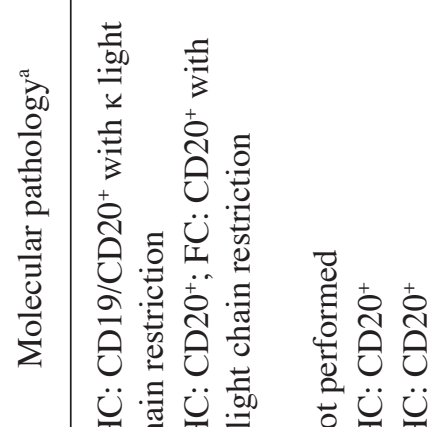

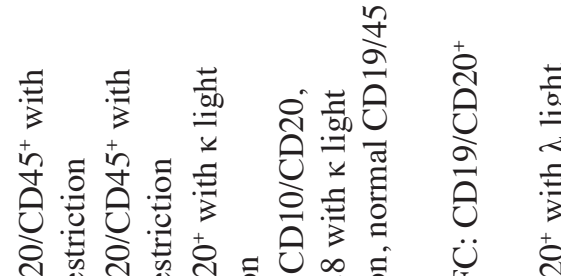

究

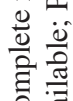

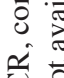

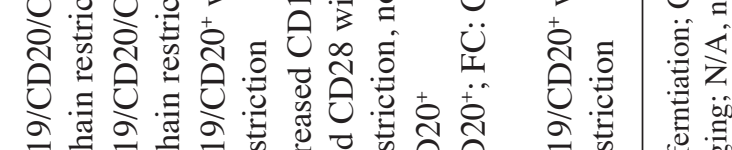

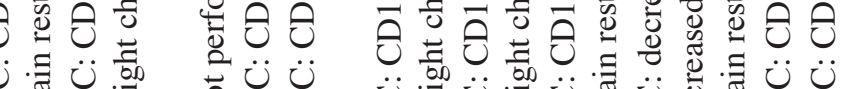

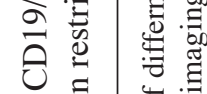

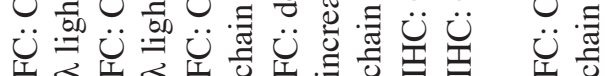

离

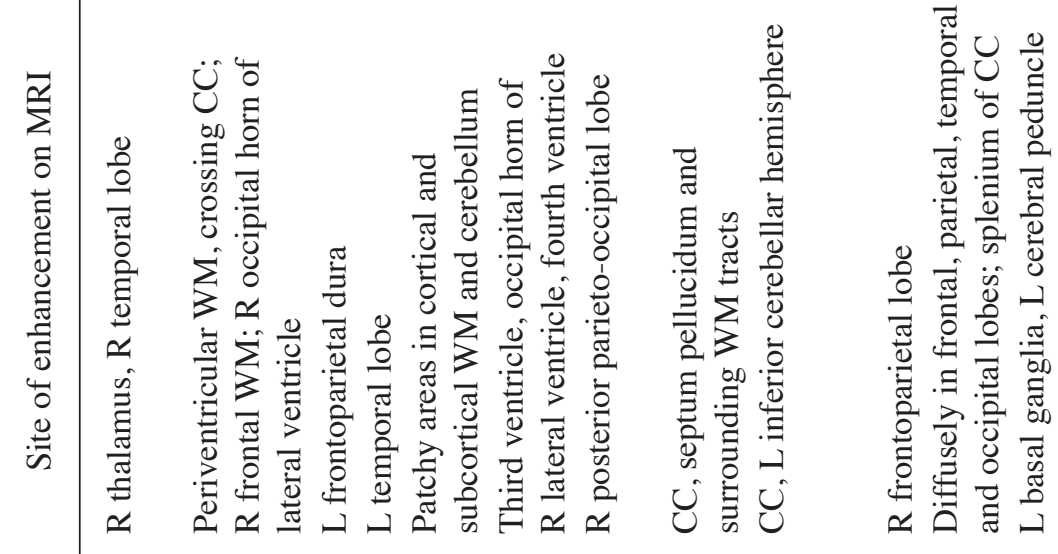

营

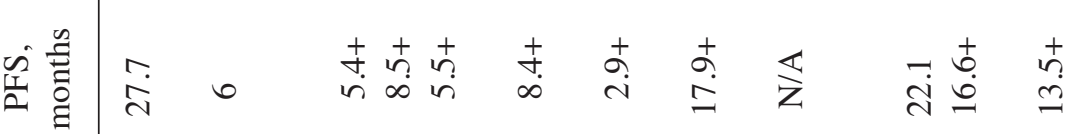

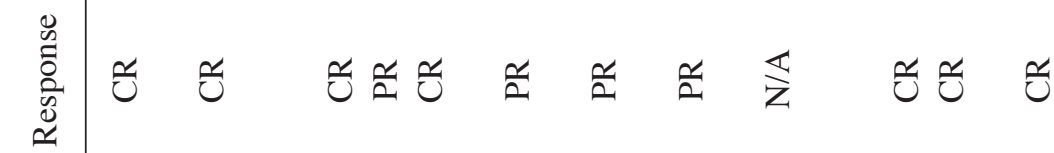

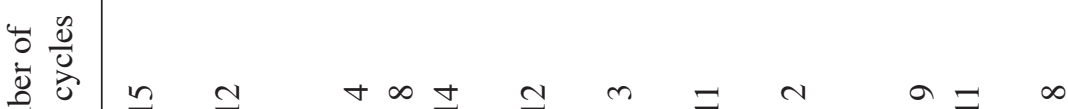

武

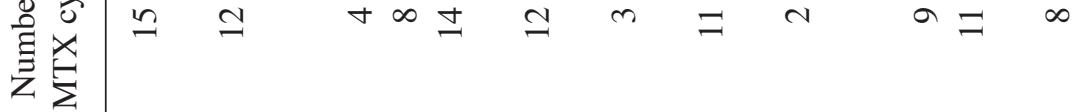

됴 है

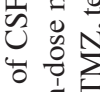

党:

品至

至若

证

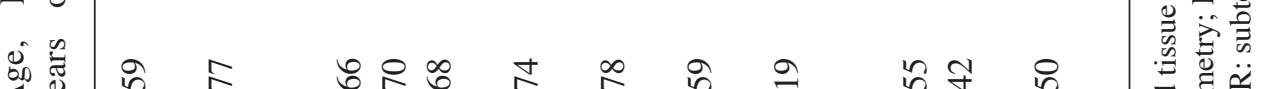

正宓 的

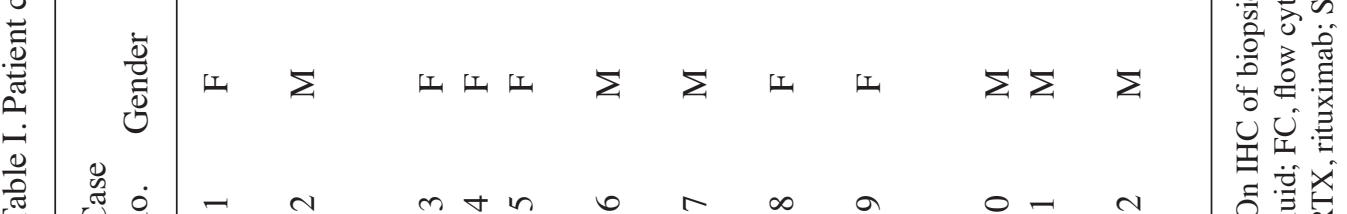




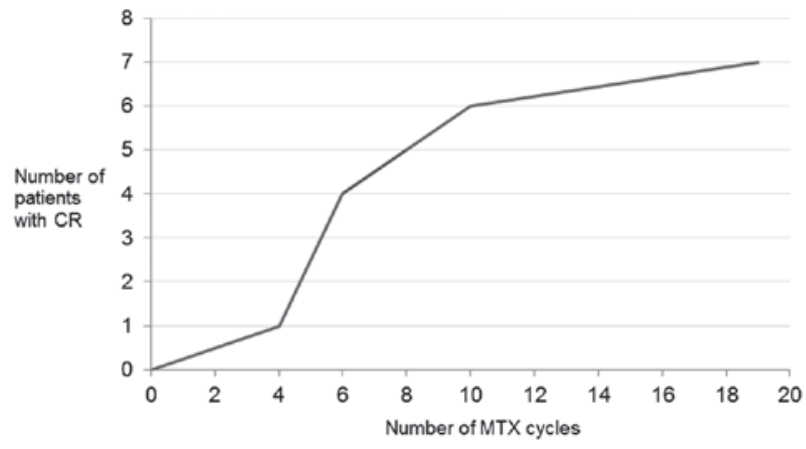

Figure 1. CR with regard to number of administered MTX cycles. The number of patients achieving CR increases with the number of MTX cycles. A minimum of four cycles of MTX had to be administered to achieve a CR. After six cycles, 4 patients had achieved a CR. CR, complete response; MTX, methotrexate.

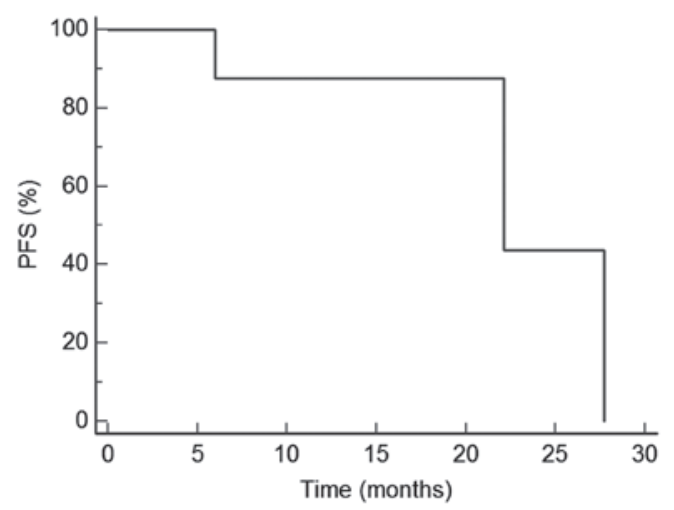

Figure 2. Kaplan-Meier estimate of the PFS of three patients (cases 1, 2 and 10) who experienced disease progression after initially achieving a complete response to high-dose methotrexate and rituximab. The median PFS time was 22.1 months. PFS, progression-free survival.

\section{Discussion}

The optimal treatment for PCNSL is unknown. Previous studies have demonstrated improved survival with combined WBRT and MTX-based therapy; however, this regimen is associated with significant risks of delayed neurotoxicity, particularly in patients $>60$ years old, manifesting as cognitive dysfunction, which can progress to dementia with gait ataxia and incontinence $(19,20)$. MTX remains the cornerstone of therapy and mounting evidence suggests that MTX-based polychemotherapy improves survival $(14,16)$. WBRT tends to be reserved for recurrent disease or those unable to receive chemotherapy.

RTX is a chimeric monoclonal antibody against the CD20 antigen on B lymphocytes, which is present in the vast majority of patients with systemic NHL and PCNSL (21). Administration of RTX improves the outcome in patients with systemic DLBCL, reduces the rate of CNS relapse in high-risk disease, and in conjunction with the $\mathrm{CHOP}$ regimen, has become a standard component of treatment for DLBCL (22). The mechanism of action for RTX includes complement-mediated and antibody-dependent cellular cytotoxicity, as well as the antibody-induced inhibition of cell growth and apoptosis (21). However, the clinical effects of RTX in PCNSL are unclear. Although RTX has poor BBB penetration due to its large molecular size, a certain degree of therapeutic drug level is achieved ( $\sim 1.5 \%$ of serum concentration) (23), likely due to disruption of the BBB by the tumor itself. This explains the rationale for using RTX in the induction phase of treatment only when BBB permeability is highest due to increased tumor burden.

To date, no randomized controlled trials of combined HD-MTX and RTX have been conducted, and treatment protocols are largely institution-dependent. However, multiple prospective and retrospective studies have indicated that the addition of RTX to MTX-based chemotherapy may improve response rates and PFS times compared with single-agent HD-MTX (Table II). In one prospective single-arm study (16), patients received induction chemotherapy with an RTX, MTX, vincristine and procarbazine regimen, and achieved an ORR of 93\% and a CRR of $78 \%$. Another study investigated combined RTX, MTX and ifosfamide therapy, and found that the addition of RTX resulted in an improved CRR (100\%) and 6-month PFS (PFS-6) rate (94\%) compared with MTX and ifosfamide alone (CRR, 68\%; PFS-6, 63\%) (15). Two previous studies evaluated combined HD-MTX and RTX $(14,24)$. Chamberlain and Johnston (14) conducted a prospective phase II trial using this regimen and reported a ORR and CRR of 80 and 60\%, respectively. These results are similar to those of the present study (ORR, 91\%; CRR, 58\%), although there were certain differences in trial design. While the patients in the present study received weekly RTX for a maximum of 8 cycles or until a radiographic CR was achieved, patients in the previous study were administered a median of 4 cycles of RTX (range, 4-6 cycles) every 2 weeks (14). It is unknown whether more frequent administration of RTX is associated with improved survival, as median OS time had not been attained in the present cohort at the time of data analysis. Notably, toxicity from RTX was limited to grade 3 or less in the patients, suggesting that additional cycles of RTX were relatively well tolerated. Holdhoff et al (24) treated 27 patients with the same combination regimen as in the present study and compared the response with that of patients who received single-agent HD-MTX at their institution. The study found that the addition of RTX resulted in an improved CRR (89\%) and PFS time (27 months). Similar to the present study, OS had not been reached in those receiving HD-MTX and RTX.

RTX may also be beneficial in individuals $\geq 65$ years of age. Treatment of this group is particularly challenging since WBRT and HD-MTX are notoriously associated with late neurotoxicity $(19,20)$. In a prospective study of 28 patients receiving RTX, HD-MTX, procarbazine and lomustine (R-MCP) (18), the ORR (82\%) and CRR (64\%) were comparable to response rates in younger subjects. OS and PFS times were also prolonged with the addition of RTX when compared with a previous single-arm study (25) using MCP alone (R-MCP group: OS, 17.5 months and PFS, 16 months; MCP group: OS, 15.4 months and PFS, 5.9 months).

Lastly, there is inconclusive data on the optimal treatment for recurrent PCNSL. The median OS time in recurrent or progressive PCNSL is $\sim 4.5$ months (26). Since the majority of patients are older ( $>60$ years) at the time of relapse, regimens with minimal toxicity are preferred. Single-agent RTX in 12 patients with recurrent PCNSL resulted in an ORR and CRR of 36 and $27 \%$, respectively, and a median OS time of 21 months (27). By contrast, data from two small retrospective 


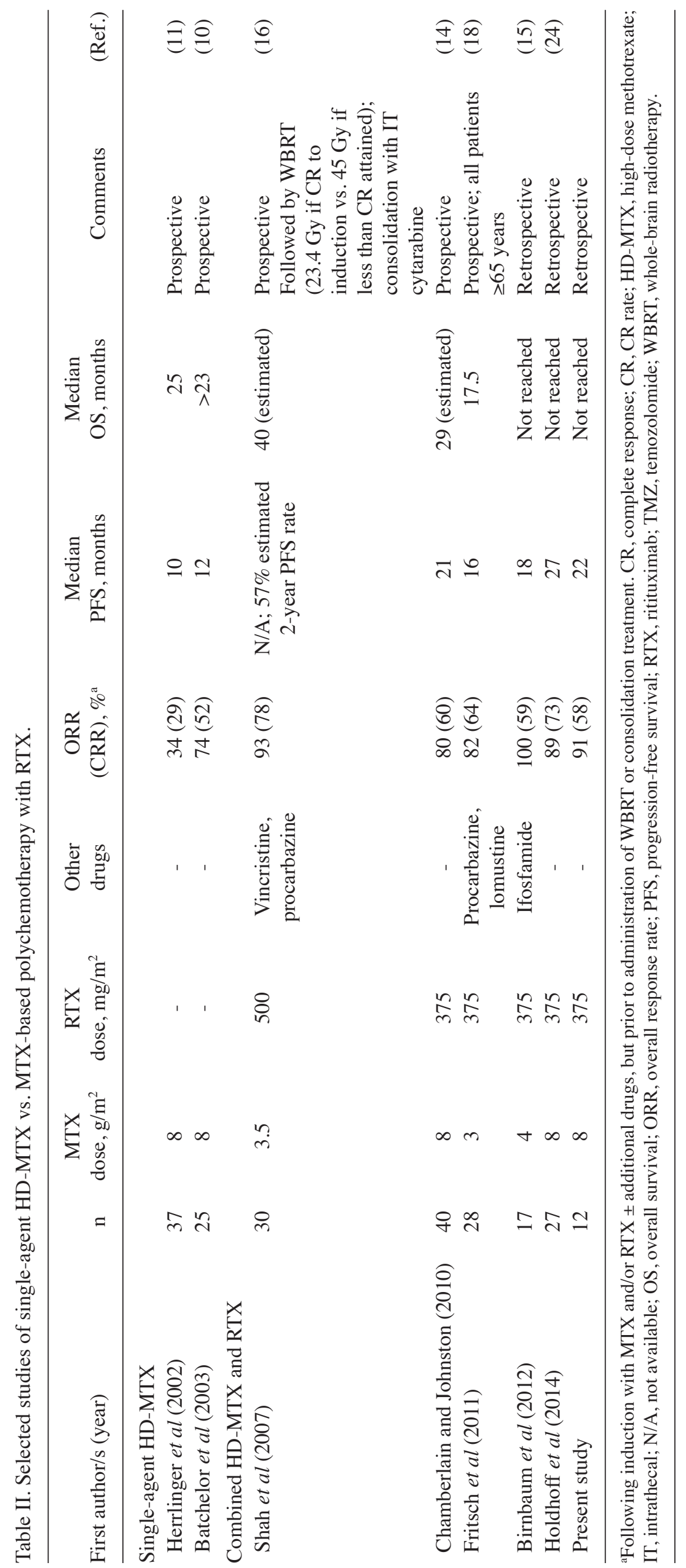


case series suggested that combined RTX and TMZ was well tolerated and resulted in a higher ORR (53-100\%), but a shorter OS time (8-14 months) $(28,29)$.

The power of the present study is limited by the small number of patients, the lack of randomized data and its retrospective nature. Nonetheless, the results support the observation that addition of RTX to HD-MTX imparts a survival benefit compared with single-agent HD-MTX. Future randomized controlled trials will provide further insight into the efficacy of RTX in PCNSL treatment. Currently, these include the HOVON 105 PCNSL/ALLG NHL24 trial (trial number, EudraCT 2009-014722-42) and the International Extranodal Lymphoma Study Group (IELSG)-32 trial (trial number, NCT01011920). The former randomizes patients to either a MTX, teniposide, carmustine and prednisolone (MBVP) regimen or a RTX + MBVP regimen, followed by consolidation with Ara-C. The IELSG-32 trial randomizes patients to either MTX + Ara-C, MTX + Ara-C + RTX or $\mathrm{MTX}+\mathrm{Ara}-\mathrm{C}+\mathrm{RTX}+$ thiotepa. It further randomizes patients who achieve a CR to any of these regimens to consolidation treatment with WBRT or HD-chemotherapy + ASCT.

In conclusion, the addition of RTX to HD-MTX in the treatment of PCNSL may increase response rates and prolong PFS times. It remains unclear whether RTX improves long-term outcome and whether the addition of other chemotherapeutic agents ameliorates survival. These questions should ideally be evaluated in prospective trials.

\section{Acknowledgements}

Preliminary results were presented as an abstract at the 10th Annual Meeting of the European Association of Neuro-Oncology.

\section{References}

1. Villano JL, Koshy M, Shaikh H, Dolecek TA and McCarthy BJ: Age, gender and racial differences in incidence and survival in primary CNS lymphoma. Br J Cancer 105: 1414-1418, 2011.

2. Mead GM, Bleehen NM, Gregor A, Bullimore J, Shirley D, Rampling RP, Trevor J, Glaser MG, Lantos P, Ironside JW, et al: A medical research council randomized trial in patients with primary cerebral non-Hodgkin lymphoma: Cerebral radiotherapy with and without cyclophosphamide, doxorubicin, vincristine and prednisone chemotherapy. Cancer 89: 1359-1370, 2000.

3. Korfel A and U Schlegel: Diagnosis and treatment of primary CNS lymphoma. Nat Rev Neurol 9: 317-327, 2013.

4. Nelson DF, Martz KL, Bonner H, Nelson JS, Newall J, Kerman HD, Thomson JW and Murray KJ: Non-Hodgkin's lymphoma of the brain: Can high dose, large volume radiation therapy improve survival? Report on a prospective trial by the radiation therapy oncology group (RTOG): RTOG 8315. Int J Radiat Oncol Biol Phys 23: 9-17, 1992.

5. Laperriere NJ, Cerezo L, Milosevic MF, Wong CS, Patterson B and Panzarella T: Primary lymphoma of brain: Results of management of a modern cohort with radiation therapy. Radiother Oncol 43: 247-252, 1997.

6. DeAngelis LM, Yahalom J, Thaler HT and Kher U: Combined modality therapy for primary CNS lymphoma. J Clin Oncol 10: 635-643, 1992.

7. Abrey LE, DeAngelis LM and Yahalom J: Long-term survival in primary CNS lymphoma. J Clin Oncol 16: 859-863, 1998.

8. Morris PG and Abrey LE: Therapeutic challenges in primary CNS lymphoma. Lancet Neurol 8: 581-592, 2009.

9. Omuro AM, Ben-Porat LS, Panageas KS, Kim AK, Correa DD, Yahalom J, Deangelis LM and Abrey LE: Delayed neurotoxicity in primary central nervous system lymphoma. Arch Neurol 62: 1595-1600, 2005.
10. Batchelor T, Carson K, O'Neill A, Grossman SA, Alavi J, New P, Hochberg F and Priet R: Treatment of primary CNS lymphoma with methotrexate and deferred radiotherapy: A report of NABTT 96-07. J Clin Oncol 21: 1044-9, 2003.

11. Herrlinger U, Schabet M, Brugger W, Kortmann RD, Küker W, Deckert M, Engel C, Schmeck-Lindenau HJ, Mergenthaler HG, Krauseneck P, et al: German cancer society neuro-oncology working group NOA-03 multicenter trial of single-agent high-dose methotrexate for primary central nervous system lymphoma. Ann Neurol 51: 247-252, 2002.

12. Thiel E, Korfel A, Martus P, Kanz L, Griesinger F, Rauch M, Röth A, Hertenstein B, von Toll T, Hundsberger T, et al: High-dose methotrexate with or without whole brain radiotherapy for primary CNS lymphoma (G-PCNSL-SG-1): A phase 3, randomised, non-inferiority trial. Lancet Oncol 11: 1036-1047, 2010.

13. Ferreri AJ, Reni M, Foppoli M, Martelli M, Pangalis GA, Frezzato M, Cabras MG, Fabbri A, Corazzelli G, Ilariucci F, et al: High-dose cytarabine plus high-dose methotrexate versus high-dose methotrexate alone in patients with primary CNS lymphoma: A randomised phase 2 trial. Lancet 374: 1512-1520, 2009.

14. Chamberlain MC and Johnston SK: High-dose methotrexate and rituximab with deferred radiotherapy for newly diagnosed primary B-cell CNS lymphoma. Neuro Oncol 12: 736-744, 2010.

15. Birnbaum T, Stadler EA, von Baumgarten L and Straube A: Rituximab significantly improves complete response rate in patients with primary CNS lymphoma. J Neurooncol 109: 285-291, 2012.

16. Shah GD, Yahalom J, Correa DD, Lai RK, Raizer JJ, Schiff D, LaRocca R, Grant B, DeAngelis LM and Abrey LE: Combined immunochemotherapy with reduced whole-brain radiotherapy for newly diagnosed primary CNS lymphoma. J Clin Oncol 25: 4730-4735, 2007

17. Wieduwilt MJ, Valles F, Issa S, Behler CM, Hwang J, McDermott M, Treseler P, O'Brien J, Shuman MA, Cha S, et al: Immunochemotherapy with intensive consolidation for primary CNS lymphoma: A pilot study and prognostic assessment by diffusion-weighted MRI. Clin Cancer Res 18: 1146-1155, 2012.

18. Fritsch K, Kasenda B, Hader C, Nikkhah G, Prinz M, Haug V, Haug S, Ihorst G, Finke J and Illerhaus G: Immunochemotherapy with rituximab, methotrexate, procarbazine and lomustine for primary CNS lymphoma (PCNSL) in the elderly. Ann Oncol 22: 2080-2085, 2011.

19. Filley CM and Kleinschmidt-DeMasters BK: Toxic leukoencephalopathy. N Engl J Med 345: 425-432, 2001.

20. Gavrilovic IT, Hormigo A, Yahalom J, DeAngelis LM and Abrey LE: Long-term follow-up of high-dose methotrexate-based therapy with and without whole brain irradiation for newly diagnosed primary CNS lymphoma. J Clin Oncol 24: 4570-4574, 2006.

21. Cang S, Mukhi N, Wang K and Liu D: Novel CD20 monoclonal antibodies for lymphoma therapy. J Hematol Oncol 5: 64, 2012.

22. Maloney DG, Grillo-López AJ, Bodkin DJ, White CA, Liles TM, Royston I, Varns C, Rosenberg J and Levy R: IDEC-C2B8: Results of a phase I multiple-dose trial in patients with relapsed non-Hodgkin's lymphoma. J Clin Oncol 15: 3266-3274, 1997.

23. Ruhstaller TW, Amsler U and Cerny T: Rituximab: Active treatment of central nervous system involvement by non-Hodgkin's lymphoma? Ann Oncol 11: 374-375, 2000.

24. Holdhoff M, Ambady P, Abdelaziz A, Sarai G, Bonekamp D, Blakeley J, Grossman SA and Ye X: High-dose methotrexate with or without rituximab in newly diagnosed primary CNS lymphoma. Neurology 83: 235-239, 2014.

25. Illerhaus G, Marks R, Müller F, Ihorst G, Feuerhake F, Deckert M, Ostertag C and Finke J: High-dose methotrexate combined with procarbazine and CCNU for primary CNS lymphoma in the elderly: Results of a prospective pilot and phase II study. Ann Oncol 20: 319-325, 2009.

26. Gerstner ER and Batchelo TT: Primary central nervous system lymphoma. Arch Neurol 67: 291-297, 2010.

27. Batchelor TT, Grossman SA, Mikkelsen T, Ye X, Desideri S and Lesser GJ: Rituximab monotherapy for patients with recurrent primary CNS lymphoma. Neurology 76: 929-930, 2011.

28. Wong ET, Tishler R, Barron L and Wu JK: Immunochemotherapy with rituximab and temozolomide for central nervous system lymphomas. Cancer 101: 139-145, 2004.

29. Enting RH, Demopoulos A, DeAngelis LM and Abrey LE: Salvage therapy for primary CNS lymphoma with a combination of rituximab and temozolomide. Neurology 63: 901-903, 2004. 